\title{
Correlation of morphological parameters with likelihood of thrombus formation in intracranial aneurysms
}

\author{
K. Shimano ${ }^{1} \&$ Y. Nakagawa ${ }^{2}$ \\ ${ }^{I}$ Department of Mechanical Systems Engineering, \\ Tokyo City University, Japan \\ ${ }^{2}$ Graduate School, Tokyo City University, Japan
}

\begin{abstract}
Accurate rupture prediction of intracranial aneurysms is crucial because not all aneurysms rupture. With past reports that aspect ratio (AR) and size ratio (SR) have significant correlations with rupture probability, these two morphological parameters are regarded as promising for rupture prediction: an aneurysm with a higher AR or SR is more likely to rupture than one with a lower AR or SR. However, $100 \%$ reliability of prediction is not guaranteed even with these parameters. It is necessary to clarify reasons for the high correlations of AR and SR with rupture status and what is responsible for unsuccessful predictions. In this paper, the authors discuss haemodynamic significance of the two parameters with a special focus on stagnant flow and resulting thrombus formation, which are considered to play important roles in the process of aneurysmal rupture. Results with computational fluid dynamics and a platelet aggregation model are also shown in order to demonstrate that stagnant flow and thrombus formation can be observed even in an aneurysm with low AR and SR.
\end{abstract}

Keywords: intracranial aneurysm, rupture prediction, thrombus formation, platelet aggregation, computational fluid dynamics.

\section{Introduction}

Intracranial aneurysms can cause fatal subarachnoid haemorrhages when they rupture. Unruptured aneurysms can be found by means of magnetic resonance imaging (MRI) or computed tomography (CT) before the patients have any symptoms, and there are neurosurgical treatments to prevent future rupture of 
detected aneurysms, e.g. clipping and coiling. However, such a treatment is not applied to every aneurysm because it is known that not all aneurysms rupture and because the treatments are so invasive that the patients' lives could be jeopardised. It is, therefore, necessary to make a critical decision about whether to apply any treatment whenever an unruptured aneurysm is found. Although it seems reasonable to treat only aneurysms with high probabilities of future rupture, it is difficult with the present understanding to predict precisely which aneurysms are likely to rupture.

Aspect ratio (AR) [1-3] and size ratio (SR) [4-6] were reported to have significant correlations with probability of aneurysmal rupture, being recognised as promising reference parameters for rupture prediction. AR is a ratio of aneurysmal dome depth to neck width while SR is defined as a ratio of dome length to parent artery diameter. It is considered that an aneurysm with a higher AR or SR is more likely to rupture than one with a lower AR or SR. Nonetheless, there is still considerable uncertainty about rupture prediction based on these two morphological parameters. For development of a more reliable rupture prediction method, it is important to elucidate what causes aneurysms to rupture.

The authors have worked on modelling of blood flow and thrombus formation in cerebrovascular bifurcation aneurysms because it is considered that slow intraaneurysmal flow and resultant thrombus formation play important roles in the process of rupture [7]. In this paper, a reason for probable mis-prediction with $\mathrm{AR} / \mathrm{SR}$ is discussed by means of theoretical and numerical analyses. Firstly, significance of the two parameters is explained with a dynamic energy balance theory. Secondly, the platelet aggregation model by Shimano et al. [8, 9] is applied to three model aneurysms, one of which was constituted from multi-slice CT images.

\section{Significance of $A R$ and $S R$}

Ujiie et al. [1] showed a significant correlation of AR with probability of rupture: almost $80 \%$ of ruptured aneurysms had ARs larger than 1.6. Other researchers such as Weir et al. [2] and Nader-Sepahi et al. [3] concurred with the correlation between AR and rupture probability. On the other hand, Dhar et al. [4], Tremmel et al. [5] and Rahman et al. [6] reported a high correlation of SR with rupture status. Tremmel et al. suggested aneurysms with SRs larger than 2.0 could have a potential risk of rupture.

Significance of AR and SR is intra-aneurysmal flow speed: the larger AR and SR become, the more slowly internal blood flows. According to the theory of Ujiie et al. [7], thrombi are considered to play an important role in degeneration of the aneurysmal wall and subsequent rupture because extremely slow blood flow and resultant thrombus formation are likely in aneurysms with high ARs. Fibrinolysis, in which thrombi are resolved, presumably causes damage to endothelia. Also Shojima et al. [10] pointed out that an extremely low level of wall shear stress (WSS) might contribute to degeneration of the artery wall. This 


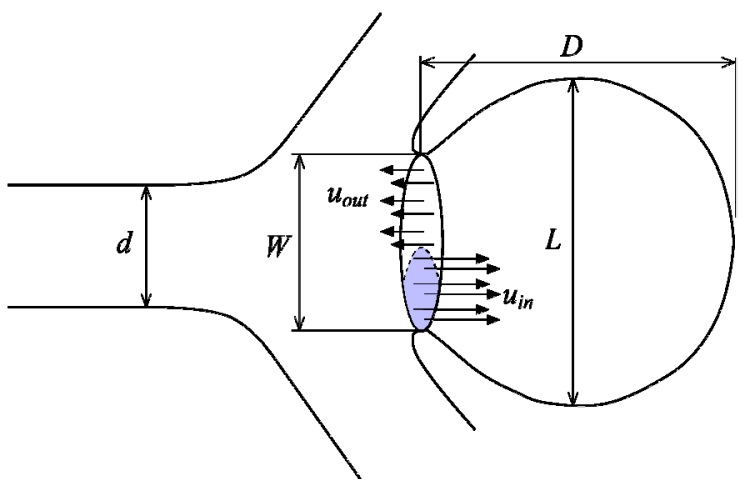

Figure 1: Hypothetical bifurcation aneurysm.

is true for aneurysms with high ARs because low WSS results from slow internal flow.

In this section, a haemodynamic reason for the high correlations of AR and SR with rupture probability is discussed. It is theoretically explained how AR and SR are associated with the intra-aneurysmal flow speed. It is also discussed why rupture prediction based on AR/SR is sometimes unsuccessful.

\subsection{Energy balance theory}

Energy conservation of blood in the hypothetical bifurcation aneurysm schematically shown in fig. 1 is considered so that magnitude of intraaneurysmal flow velocity can be approximately estimated. $L, D$ and $W$ respectively represent lateral length, depth and neck width of the aneurysm. AR= $D / W$ and $\mathrm{SR}=D / d$ where $d$ is parent vessel diameter. Deformation of the artery wall is neglected because results of past numerical studies (e.g. Torii et al. [11]) imply the rigid wall assumption does not cause dramatic change in the velocity magnitude. Effects of unsteadiness are also negligible because velocity averaged over the pulse period is considered here.

There are three fluid-dynamical mechanisms which affect the amount of energy kept in the dome: (a) convective energy transport by incoming flow velocity $u_{\text {in }}$ which brings energy into the dome, (b) convective energy transport by outgoing flow velocity $u_{\text {out }}$ which takes energy out of the dome and (c) energy loss caused by wall shear stress. All the three contributions should balance out: energy in the aneurysmal dome is increased by (a) and decreased by (b) and (c).

As shown in Fig. 1, the cross section at the neck is divided into the in-flow and out-flow regions, and $u_{i n}$ and $u_{\text {out }}$ stand for averaged velocity components perpendicular to the cross sectional plain in the respective regions. On the assumption that inflation of the aneurysmal dome is negligible, the two velocity components satisfy the mass conservation law expressed by

$$
A_{\text {in }} u_{\text {in }}=A_{\text {out }} u_{\text {out }}
$$


where $A_{\text {in }}$ and $A_{\text {out }}$ represent in-flow and out-flow areas, respectively. Here, ratio $\kappa$ is defined so that $A_{\text {in }}$ and $A_{\text {out }}$ can be calculated from the total cross sectional area $A$ :

$$
A_{\text {in }}=\kappa A \quad, A_{\text {out }}=(1-\kappa) A .
$$

The amount of energy brought into the dome through $A_{\text {in }}$ per unit time is evaluated by

$$
E_{\text {in }}=A_{\text {in }} u_{\text {in }}\left(\frac{\rho V_{\text {in }}^{2}}{2}+p_{\text {in }}\right)
$$

where $V_{\text {in }}$ and $p_{\text {in }}$ are flow speed and pressure averaged over the in-flow area. The amount of energy taken out of the dome through $A_{\text {out }}$ per unit time can be calculated in the same manner:

$$
E_{\text {out }}=A_{\text {out }} u_{\text {out }}\left(\frac{\rho V_{\text {out }}^{2}}{2}+p_{\text {out }}\right) \text {. }
$$

Angles formed by flow directions and the cross-sectional plain at the bottleneck, where the flow accelerates, are unlikely to deviate greatly from 90 degrees. Thus, we assume that $V_{\text {in }} \approx u_{\text {in }}$ and $V_{\text {out }} \approx u_{\text {out }}$. It is also assumed that $p_{\text {in }} \approx p_{\text {out }}$. These approximations and eqns. (1)-(4) lead to the following expression for energy change due to convection:

$$
E_{\text {in }}-E_{\text {out }} \approx \rho A K u_{\text {in }}^{3} \quad \text { where } \quad K=\frac{\kappa(1-2 \kappa)}{2(1-\kappa)^{2}} .
$$

On the other hand, energy loss caused by WSS should agree with frictional work per unit-time $W_{\text {wall }}$, which can be approximated by

$$
W_{\text {wall }} \approx S \tau_{\text {wall }} U
$$

where $S, \tau_{\text {wall }}$ and $U$ stand for area of the arterial wall surface within the dome, wall shear stress averaged over $S$ and representative velocity within the aneurysmal dome. Equation (6) is rewritten into

$$
W_{\text {wall }} \approx \mu D U^{2} \text {. }
$$

using the following approximations to $S$ and $\tau_{\text {wall }}$

$$
\begin{gathered}
S \approx L D \\
\tau_{\text {wall }} \approx \frac{\mu U}{L}
\end{gathered}
$$

where $\mu$ is blood viscosity. As $E_{\text {in }}, E_{\text {out }}$ and $W_{\text {wall }}$ are in equilibrium, eqns. (5) and (7) result in the following expression of energy balance:

$$
\rho A \overline{K u_{i n}^{3}} \approx \mu D \overline{U^{2}} .
$$


Lines over quantities in eqn. (10) mean that the quantities are averaged over the pulse period. Although $\overline{a b}=\bar{a} \cdot \bar{b}$ does not hold strictly true, the approximation $\overline{a b} \approx \bar{a} \cdot \bar{b}$ is still reasonable when only the scale of each quantity is concerned. It is also assumed that the magnitude of the incoming flow velocity $u_{i n}$ is comparable to the mean velocity of the main flow $u$. In addition, $A \approx W^{2}$ is obvious. Application of these approximations to eqn. (10) results in

$$
\frac{\bar{U}}{\bar{u}} \approx \sqrt{\frac{\rho W^{2} \bar{K} \cdot \bar{u}}{\mu D}} .
$$

The left hand side of eqn. (11) represents ratio of the intra-aneurysmal flow speed $U$ to the main flow speed $u$. The degree of flow stagnancy in the dome can be evaluated by this ratio. After some arrangements, the flow speed ratio can be associated with AR and SR:

$$
\frac{\bar{U}}{\bar{u}} \propto \sqrt{\frac{\mathrm{Re}}{A R}} \quad \frac{\bar{U}}{\bar{u}} \propto W \sqrt{\frac{\overline{\frac{\tau_{m v}}{S R}}}{}}
$$

where $\mathrm{Re}$ is the Reynolds number based on the neck width and $\tau_{m v}$ is wall shear stress of the mother vessel approximated by $\tau_{m v} \propto \mu u / d$. Note that $\tau_{m v}$ does not change dramatically in normal cerebral arteries.

The relations in (12) clearly show that both AR and SR correlate with the degree of flow stagnancy. Although there are still other influential factors such as Re and $\mathrm{W}$, it can be concluded that the intra-aneurysmal flow tends to be slower when AR or SR is larger. This is considered to be one of the primary reasons for high correlations of these parameters with rupture probability.

\subsection{Limit to energy balance theory}

In the energy balance theory, each basic physical quantity in the dome is represented by a single scale. For example, only $U$ is used to represent the intraaneurysmal velocity scale. This implies that fairly simple internal flow is assumed. However, in some conditions, internal flow is so complicated that the relations in eqn. (12) are not valid any more. A typical example of such complicated flow can be found in the case where flow separation occurs. In the presence of flow separation, flow speed in the separation bubble is much lower than in other regions and, consequently, WSS exerted on the separation bubble area is much lower than the estimation with eqn. (9). Another example of complicated intra-aneurysmal flow can be observed in a bulky aneurysm where kinetic energy brought by the incoming flow cannot spread fully within the aneurysmal dome. A lack of uniformity can cause slowdown in flow speed in a limited part of the dome. It should be emphasised that even "local" stagnancy of the intra-aneurysmal flow could induce thrombus formation and might lead to rupture. Rupture prediction based on AR/SR could be unsuccessful because AR and SR cannot represent any reduction in "local" flow speed caused by flow 
separation or large aneurysmal volume: they are "global" parameters only accounting for overall morphological features.

Computational fluid dynamics (CFD) allows us to detect any "local" flow separation and slowdown in flow speed if spatial resolution of the computational grid is fine enough. This is one of the reasons why numerical simulation is regarded as a promising tool in this field.

\section{Modelling of intra-aneurysmal flow and platelet aggregation}

CFD and the platelet aggregation model by Shimano et al. [9] were applied to three model aneurysms so as to demonstrate that the "local" stagnancy discussed in Section 2.2 makes it impossible to estimate the internal velocity scale or the degree of thrombus formation from AR/SR.

The three model aneurysms shown in figs. 2-4 are named Bulky, Slim and Real Models, respectively. The maximum Reynolds number, Womersley parameter, AR and SR of each model are listed in Table 1.

Table 1: Haemodynamic and morphological parameters of the three model aneurysms.

\begin{tabular}{|c|c|c|c|c|}
\hline Model & $\begin{array}{c}\text { Max.Reynolds } \\
\text { number }\end{array}$ & $\begin{array}{c}\text { Womersley } \\
\text { parameter }\end{array}$ & Aspect Ratio & Size Ratio \\
\hline Bulky & 485 & 2.39 & 2.3 & 3.2 \\
\hline Slim & 728 & 2.25 & 2.0 & 1.6 \\
\hline Real & 797 & 3.36 & 0.9 & 1.1 \\
\hline
\end{tabular}

Bulky Model shown in fig. 2 is identical to the glass aneurysm experimentally tested by Takahashi et al [12]. They reported that thrombus formation was clearly observed in the glass aneurysm when it was embedded into a canine artery. Bulky Model has a larger SR than the other models because the mother vessel diameter $d$, which was presumably determined according to the canine artery, is smaller. Slim Model shown in fig. 3 is smaller in lateral length of the dome than Bulky Model, although ARs of the two models are comparable. More details of Bulky and Slim Models are available in Reference [13].

The aneurysm in Real Model shown in fig. 4 is located across the whole anterior communicating artery and regarded as an aneurysm with a low risk of rupture because it has an AR of 0.9 and SR of 1.1, each of which is much smaller than the corresponding criterion.

Real Model was constituted from multi-slice CT images of a real aneurysm. Constitution procedures of the 3-D computational model are depicted in fig. 5. In the beginning, a 3-D polygon model was made up by layering 184 CT images. At this point, two separate arteries were erroneously recognised as adhering to each other. The operator needed to do time-consuming manual work to correct this error using Rhinoceros and other software. More sophisticated software should be developed for reduction in the workload. 


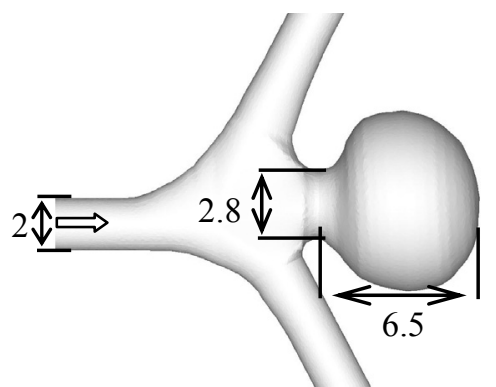

Figure 2: Bulky model.

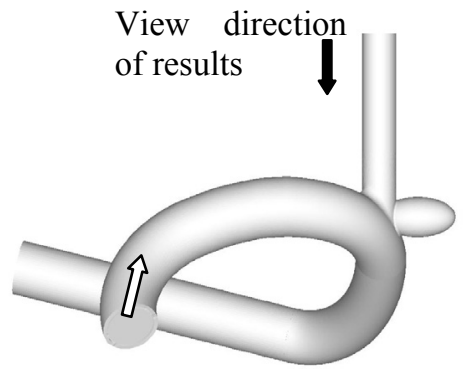

Figure 3: $\quad$ Slim model.

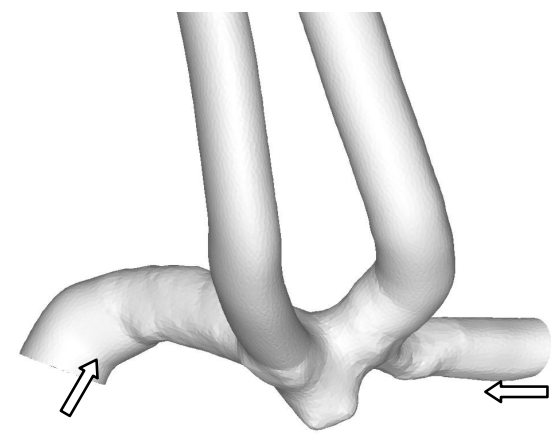

Figure 4: Real model.

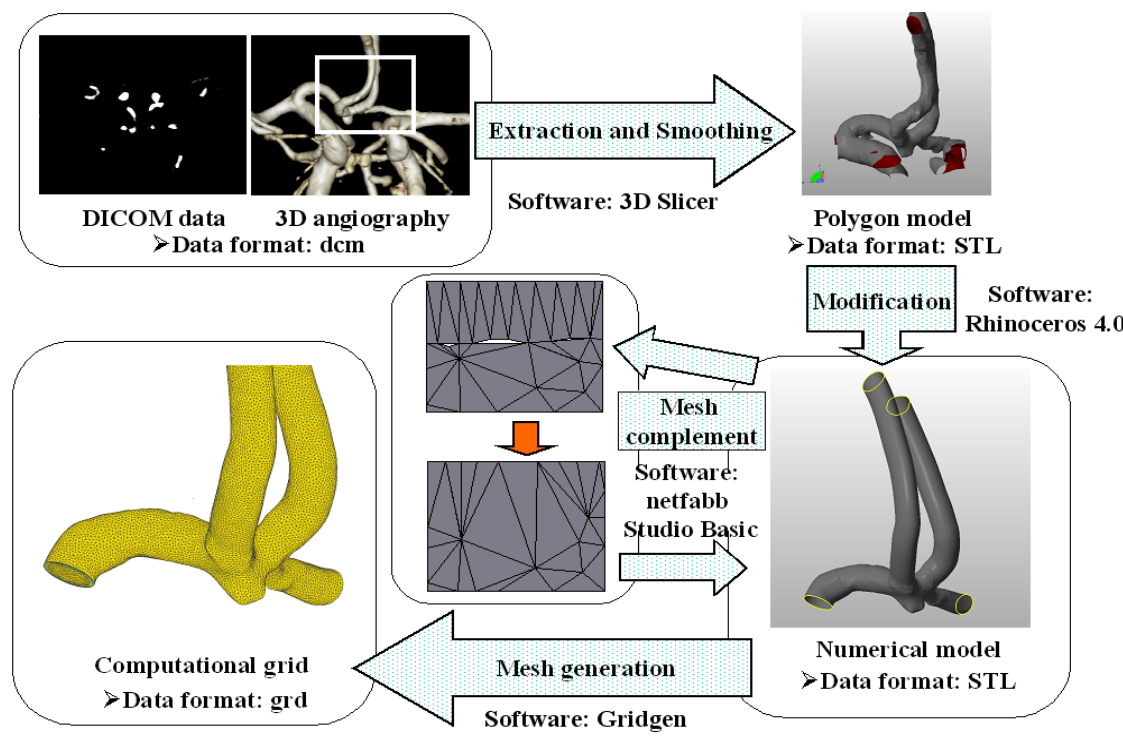

Figure 5: Procedures to constitute a computational model from CT images. 
The unsteady incompressible Navier-Stokes equations and continuity equation were numerically solved. Blood was assumed to be a Newtonian fluid and flow was treated as laminar. It has been shown that the non-Newtonian effect of blood does not change significantly the intra-aneurysmal velocity distribution [13].The cell-centred finite volume approach was used for spatial discretisation of the governing equations. Unstructured grid systems were employed so that the complicated geometry of each model could be properly expressed. The SIMPLE algorithm was used for coupling of pressure and velocity. It was confirmed that the authors' CFD code could produce flow patterns in Bulky model similar to those observed in the experiment of Takahashi et al. [12].

When the platelet aggregation model [9] was applied, flow velocity components calculated by CFD were used to evaluate transport by convection. In the aggregation model, transport equations were solved for three parameters: density of primarily aggregated platelets $N_{1}$, that of secondly aggregated platelets $N_{2}$ and concentration of adenosine diphosphate (ADP) $n_{A}$. No effect of adhesion of platelets to artery walls or coagulation was taken into consideration.

ADP released from haemolysed red blood cells (RBCs) is considered to activate platelets and induce aggregation [13]. It was assumed in the present simulation that $20 \%$ of ADP contained in RBCs impinging against the wall was released into plasma in the region within a $1 \mathrm{~mm}$ radius of the stagnation point. The impingement of the main flow is explained in Section 4.1.

\section{Computational results}

\subsection{Flow patterns}

Velocity vectors on the central plains of Bulky and Slim Models are compared in figs. 6 and 7 at the temporal point of the systole. The view direction of fig. 7 is shown in fig. 3 with a dark arrow. A reference arrow in each figure represents $50 \%$ of the maximum mean velocity of the main flow at the inlet.

It is observed in each model that the incoming main flow hits the protruding wall of the neck at the entrance to the dome and that some blood moves into the dome after the impingement against the neck. However, in Bulky Model, stagnant blood occupies a large part of the dome while low speed flow is only observed near the apex of the aneurysmal dome in Slim Model. This sharp contrast between flow patterns in the two models might be partly attributable to the larger SR of Bulky Model. It is, however, apparent from fig. 6 that a lack of flow uniformity caused by the too large volume of the dome is responsible: kinetic energy brought into the dome cannot spread fully within the dome. In such flow conditions, the estimation based on eqn. (12) is no longer valid. For this reason, flow patterns in the two models with comparable ARs are significantly different.

Velocity vectors in Real Model are depicted in fig. 8 at the systole and diastole. Incoming flow hits the wall near the entrance to the dome and some blood circulates inside before going out of the dome. This flow pattern in Real Model seems similar to that in Slim Model but the magnitude of flow speed in 
Real Model is larger even near the deep end of the aneurysmal dome. The higher flow speed in the circulating flow in Real Model obviously results from smaller $\mathrm{AR}$ and SR.

The most important observation in fig. 8 is a small separation bubble at the apex of the dome. This separation bubble, in which flow speed is much lower than in the circulating flow, exists throughout the pulse cycle and is likely to give a good chance of thrombus formation. This stagnant flow in the small region cannot be detected by AR or SR as these two parameters are much smaller than respective criteria: $\mathrm{AR}=0.9(<1.6)$ and $\mathrm{SR}=1.1(<2.0)$.

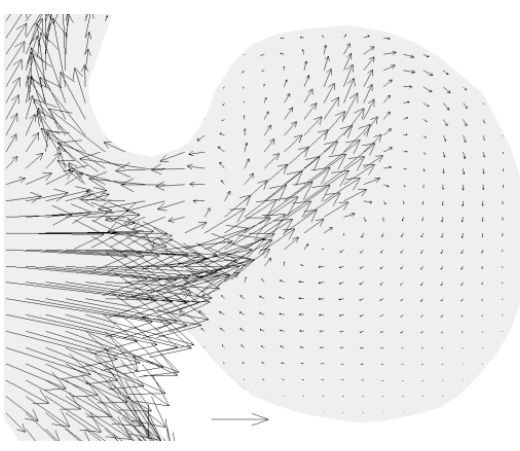

Figure 6: Velocity vectors on the central plain in bulky model at the systole.

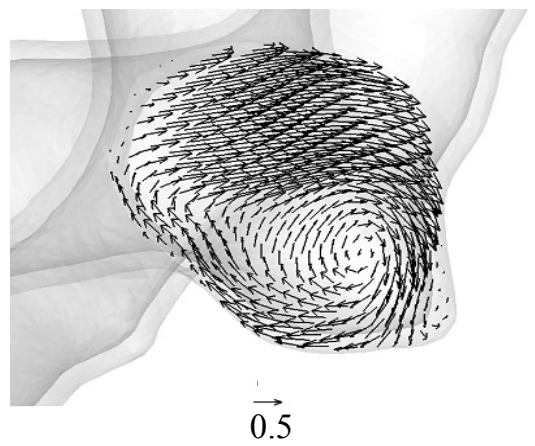

(a) Systole.

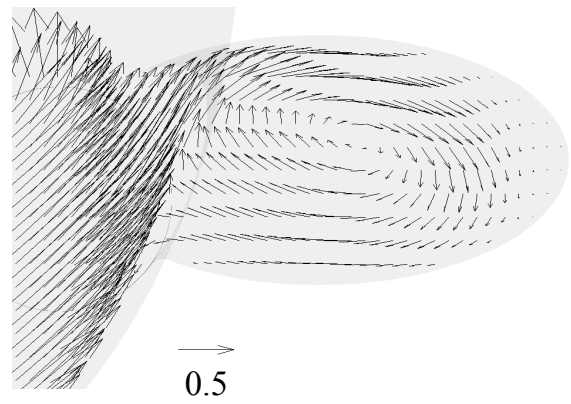

Figure 7: Velocity vectors on the central plain in slim model at the systole.

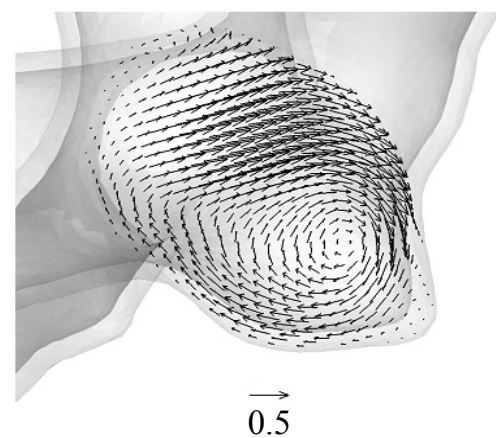

(b) Diastole.

Figure 8: $\quad$ Velocity vectors in real model at the systole and diastole.

\subsection{Platelet aggregation}

This section refers to density of primarily aggregated platelets $N_{1}$, which was calculated by the platelet aggregation model. Here, density means the number of platelets per volume. For simplicity, $N_{1}$ is expressed as a percentage to the total 
density of platelets contained in normal blood. Time $t$ is also represented in a dimensionless being divided by the pulse period $T$.

Figure 9 shows instantaneous distribution of $N_{1}$ along the aneurysmal wall in Real Model at $t / T=11.8$. The location of the highest $N_{1}$ is near the apex of the aneurysmal dome where the flow separation discussed in the previous section occurs. The low-speed flow in the separation bubble seems responsible for the highest $N_{1}$ because slow flow enables a long residence time of ADP which is necessary for active platelet aggregation.

0 0.2 0.4

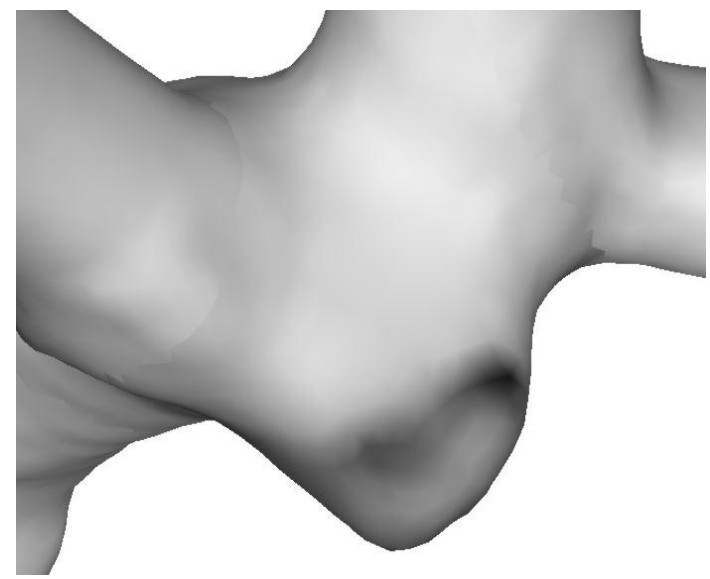

Figure 9: Instantaneous distributions of $N_{1}$ (density of primarily aggregated platelets) in the vicinity of the wall surface in real model at $t / T=11.8$.

Figure 10 shows temporal changes in $N_{1}$ at the points where the maximum values of $N_{1}$ in the respective models are found at $t / T=11.8$. At the observation point in Bulky Model, aggregation starts after $t / T=2.0$ because it takes ADP two times the pulse period to move from the location of haemolysis to the observation point. A monotone increase in $N_{1}$ observed after the start of aggregation is fairly quick due to the extremely slow internal flow shown in fig. 6. On the other hand, cyclic changes in $N_{1}$ are found at the observation points in Slim and Real Models. Reduction in $N_{1}$ occurs at the systole because some aggregated platelets are flushed out by high speed flow at the systole. Despite the drop in $N_{1}$, aggregated platelets stay near the observation points throughout the pulse cycle, keeping $N_{1}$ higher than $0.5 \%$ in Slim Model and approximately $0.1 \%$ in Real Model.

No effect of adhesion is included in the present aggregation model. However, adhesion of activated platelets to aneurysmal walls actually occurs because Tanahashi et al. [14] confirmed that activated platelets adhere to endothelia. The 


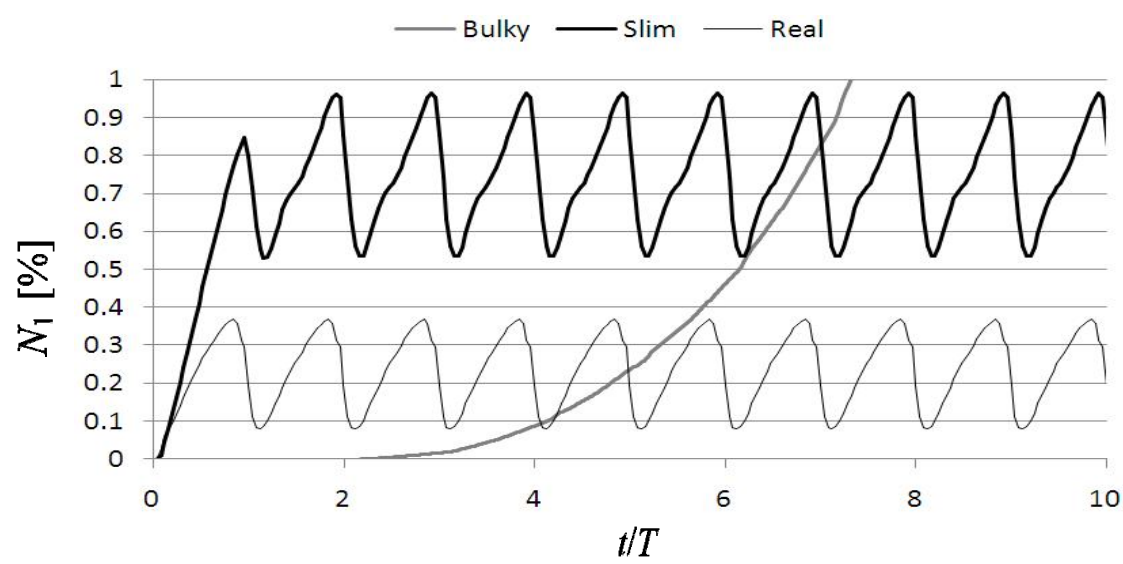

Figure 10: Temporal changes in $N_{1}$ (density of primary-aggregated platelets) at the points where the maximum values of $N_{1}$ in respective models are observed at $t / T=11.8$.

number of platelets adhering to the wall is, therefore, considered to keep increasing near the observation points in Slim and Real models. As platelets adhering to endothelia contribute to acceleration of the following coagulation, it seems a natural conclusion that probability of thrombus formation is comparably high in both models.

\section{Conclusions}

It is theoretically shown that AR and SR are linked with flow speed in aneurysms. This is presumably a reason for high correlations of the two parameters with rupture probability. However, the present numerical results demonstrate that AR and SR do not always reflect intra-aneurysmal flow status and, presumably, likelihood of thrombus formation. Although Bulky and Slim Models have comparable ARs, stagnant flow occupies a larger region of the dome in Bulky Model and platelet aggregation in the dome is more active. Real Model with much smaller AR and SR seems to have a good chance of thrombus formation due to flow separation. These findings might account for uncertainty about rupture prediction based on AR and SR. As the degree of thrombus formation is determined by not only stagnant flow or platelet aggregation, further studies are necessary for more detailed insights into intra-aneurysmal thrombus formation with adhesion and coagulation taken into account.

\section{Acknowledgement}

The authors thank Cybernet Systems Co., Ltd. for providing CT data. 


\section{References}

[1] Ujiie, H., Tamano, Y., Sasaki, K. \& Hori, T., Is the aspect ratio a reliable index for predicting the rupture of a saccular aneurysm? Neurosurgery, 48(3), pp.495-503, 2001.

[2] Weir, B., Amidei, C., Kongable, G., Findlay, J.M., Kassell, N.F., Kelly, J., Dai, L. \& Karrison, T.G., The aspect ratio (dome/neck) of ruptured and unruptured aneurysms, Journal of Neurosurgery, 99(3), pp.447-451, 2003.

[3] Nader-Sepahi, A., Casimiro, M., Sen, J. \& Kitchen, N.D., Is Aspect Ratio A reliable Predictor of Intracranial Aneurysm Rupture? Neurosurgery, 54(6), pp.1343-1348, 2004.

[4] Dhar, S., Tremmel, M., Mocco, J., Kim, M., Yamamoto, J., Siddiqui, A.H., Hopkins, L.N. and Meng, H., Morphology Parameters for Intracranial Aneurysm Rupture Risk Assessment, Neurosurgery, 63(2), pp.185-197, 2008.

[5] Tremmel, M., Dhar, S., Levy, E.I., Mocco, J. and Meng, H., Influence of Intracranial Aneurysm-to-Parent Vessel Size Ratio on Hemodynamics and Implication for Rupture: Results from a Virtual Experimental Study, Neurosurgery, 64(4), pp.622-631, 2009.

[6] Rahman, M., Smietana, J., Hauck, E., Hoh, B., Hopkins, N., Siddiqui, A., Levy, E.I., Meng, H. and Mocco, J., Size Ratio Correlates With Intracranial Aneurysm Rupture Status (A Prospective Study), Stroke, 41, pp.916-920. 2010.

[7] Ujiie, H., Tachibana, H., Hiramatsu, O., Hazel, A.L., Matsumoto, T., Ogasawara, Y., Nakajima, H., Hori, T., Takakura, K. \& Kajiya, F., Effects of size and shape (aspect ratio) on the hemodynamics of saccular aneurysms: a possible index for surgical treatment of intracranial aneurysms, Neurosurgery, 45(1), pp.110-130, 1999.

[8] Shimano, K., Hayashi, T., Ujiie, H., Ono, T. \& Enomoto, Y., Modelling of platelet aggregation in aneurysm, Proc. of 7th Int. Conf. on Modelling in Medicine and Biology, WIT Press, Southampton, pp.43-52, 2007.

[9] Shimano, K et al. (2009) Modeling of platelet aggregation in cerebrovascular bifurcation aneurysms, IFMBE Proc. vol. 25, World Congress on Medical Physics\& Biomedical Engineering, Springer, pp.495498, 2009.

[10] Shojima, M., Oshima, M., Takagi, K., Torii, R., Hayakawa, M., Katada, K., Morita, A. and Kirino, T., Magnitude and Role of Wall Shear Stress on Cerebral Aneurysms -Computational Fluid Dynamics Study of 20 Middle Cerebral Artery Aneurysms-, Stroke, 35, pp.2500-2505, 2004.

[11] Torii, R., Oshima, M., Kobayashi, T., Takagi, K. and Tezduyar, T.E., Influence of Wall Elasticity on Image-Based Blood Flow Simulations (in Japanese with English abs.), Transactions of Japan Society of Mechanical Engineers, Series A, 70(697), pp.1224-1231, 2004.

[12] Takahashi, N., Ujiie, H., Yotoriyama, T., Suzuki, Y., Hori, T. \& Kaibara, M., Flow visualization study of the endothelialized glass aneurysm model 
implanting canine carotid artery (in Japanese with English abs.), Journal of Japanese Society of Biorheology, 18(4), pp.143-148, 2004.

[13] Shimano, K., Kudo, T., Yoshimoto, S., Ujiie, H. and Enomoto, Y., A numerical study of blood flow patterns in cerebral aneurysms and the causal relationship with platelet aggregation. International Journal of Design \& Nature and Ecodynamics, 5(2), pp.122-141, 2010.

[14] Tanahashi, N., Fukuuchi, Y., Tomita, M., Yokoyama, M., Tomita, Y., Inoue, K. and Schiszler, I., Platelet adhesion to human brain microvascular endothelial cells in vitro (Observation with video-enhanced contrast microscopy), Neuroscience Letters, 274, pp.199-202, 1999. 